\title{
Alternativas de crecimiento económico: una introducción al análisis de la micro-empresa
}

\author{
Massimo de Franchi
}

\section{El sector Informal}

El sector informal es aquel sector de la producción nacional de la economia salvadorena donde encontramos las micro-empresas. Vamos entonces a dar un paseo por este mundo de la producción económica.

En los paises industrializados el conjunto de las pequenas y medianas empresas ha sido siempre considerado como el sector de la producción artesanal. Por la homogeneidad interna del crecimiento endógeno, este lejido ha producido en el siglo diecisiete y dieciocho varias empresas que se ha elevado a grandes monopolios en este siglo. El mito napoleónico que lodo soldado tiene el basión de general en su mochila era todavia realidad.

En los países económicamente dependientes que poco a poco salieron de las condiciones de colonias lambién se fue creando este sector "pobre" de la actividad productiva y de servicios. Las memorias de $\mathrm{Mi}$ guel Mármol nos ilustran aquel sector de las arlesanías, zapateros, carpinteros y olros menesteres de la economía salvadorefia. Sin embargo aquellos artesanos crecieron en la sombra de los oligopolios acaparadores de la casi lotalidad del ahorro interno que nunca les permitieron realizar el sueño "napoleónico". Aquellos artesanos absorbieron todas las crisis ciclicas del sistema ya mundializado y vinieron creando un sector de productores pobres de bienes y servicios, sin o con poca calificación profesional considerado por los economistas como el refugio 
del cada vez más grueso "ejército indusirial de reserva" de los paises "económicamente dependientes". Inclusive la teoría económica "clásica" no consideraba este conjunto como "un sector económico", identificándolo como el sector de la "subsistencia urbana" al igual que en la agricultura, el sector de los minilundistas.

La expresión "sector informal" ha sido moldeada por una "serie de esludios sobre los sectores pobres de la producción en Kenya y en Colombia a principios de la década de los anos 70 , llegando asi a producir un concepto, aunque con contenidos todavla restrictivos que permitiera describir este mundo de la producción. Estos dos estudios llegaron a la descripción siguiente de este "sector".'

a. las operaciones del sector informal son mas labor- using por unidad de producción que el seclor lormal.

b. pero el crecimiento de los ingresos en el sector inlormal implica necesariamente un cambio de la estructura de la demanda en lavor del sector informal mismo;

c. esto porque la producción del sector informal se destina a la demanda de los pobres, mientras que los productos del sector formal encuenlran su público en los sectores ricos de la población;

d. de esto viene que los Gobiemos deberian impulsar las compras direclas o por medios de empresas del sector formal de la producción obtenidas en el olro. Tendrian también que promover la inivestigación sobre técnicas que puedan ser utilizadas por el sector informal. Y todo eslo por medio de una adecuada política de repartición sectorial y regional.

Es así que por medio de estos dos estudios de la OIT se llegaba por primera vez a una descripción de este sector como parte del sistema productivo global y se le atribuía un rol y una demanda especifica: "el sector de los pobres". Consecuentemenle se le atribuyó también una tecnologia propia es decir una especilicidad en la organización de los factores de producción, capacidades técnicas e instrumentos de producción. Estos estudios propusieron también un esquema de relaciones con el sector "Yormal" de la producción industrial y de servicios.

Se sabe que la acumulación del bienestar [whealth] entre la parte mas estrecha del sector moderno es una consecuencia de la concentración del poder político en este seclor provocando un empobrecimiento y.

1. Employment, Income and Equality: a strategy for increasing productive employment in Kenya, ILO, Geneva 1972. 
une depresión económica del otro sector, el informal. Todo el problema está en el crecimienlo muy lento de los altos ingresos que han dejado crecer en los "slums" o barriadas o colonias marginales, grupos de "requirientes de empleo" como periferias literal y figurativamente, de bajos ingresos. La dicotomia entre los dos grupos se reflejan en la relación con el aparato estatal y en el modelo de distribución espacial de las urbanizaciones.

Enirando en las funciones esiructurales del sector formal de la producción de bienes y servicios se observa que este tiene un acceso directo al crédito, a las Iramilaciones con el exterior, a los permisos de trabajo para los técnicos extranjeros, y a una lisla formidable de otros beneficios que favorecen la reducción de costos del capital en relación al costo de mano de obra. $Y$ además se beneficia de muchas de las restricciones de la competencia en contra de las economias exteriores. Este sector se caracteriza por sus grandes empresas. su tecnología solisticada, sus elevados salarios, sus elevadas ganancias y la presencia de la propiedad extranjera, que relaciona la economía local con las economías industrialmente dominantes.

El sector informal por su parte, se encuentra mas en seguida ignorado, a veces ayudado, y en seguida perseguido por las autoridades. Las empresas del sector, la mayoria individuales, no tienen acceso a las instiluciones formales del crédito y las fuentes más importantes de tecnologia extranjera. Muchas empresas tienen que actuar atuera de la legalidad... lo cual se debe a una limitación oficial de acceso para obtener el derecho de actuar aumentando el riesgo y la incertidumbre de mantener su nivel de vida ya muy bajo.

Este análisis se aplica lambién al mismo sector agrícola... en esta situación el empresario del seclor informal debe luchar constantemente para protegerse de todos los riesgos, y por eso se encuentra siempre cauteloso frente a la innovación. particularmente de la agricultura donde el no puede ir en contra de ninguna perdida.

En El Salvador la desigual absorción del progreso técnico, junto a las particularidades de la prevaleciente estructura de la demanda y las esinucturas oligopólicas del mercado han generado una estructura de la producción particularmenle heterogenea. Tal estructura incluye por una parte unidades económicas que operan según un modo de producción relativamente intensivo de capital, mientras otras producen mediante formas mas intensiva de Irabajo. Las primeras constiluyen el dicho "seclor moderno" de la economia, mientras que las segundas, el "sector informal". 
Los productos del sector Informal se encuentran como complemento, en competencla o como substituto temporáneo de los del sector moderno. Su clientela se diferencia en principio por las categorias de ingreso aunque no siempre es asl en muchos servicios. El sector intormal provee con una producción de bienes y servicios muy importante y esencial al desarrolio a la industrial nacional, con mano de obra, bienes y servicios. Aunque la teoria clásica opine que este sector no produce ningún bien ni servicio "economico" olvidando los efectos de interrelaciones y de substitución de importaciones que este sector esconde. Como este es el caso, determinando la elección de los productos a producir en función de la redistribución del ingreso, hay campo económico para establecer interrelaciones con óplimos resultados linancieros.

La fuerte dependencia del sector informal impone que cualquier incremento de las productividades del sector moderno provoquen un incremento del empleo en el sector informal y por otro lado los incre. mentos de los sectores están todos estrechamente relacionados con las pautas de cambios tecnológicos del sector externo.

En seguida la división del Irabajo en estas empresas es muy limilada. Muchas están organizadas como empresas familiares y emplean a parientes como ocupados, y formas tradicionales de relaciones laborales. Existe en el sector una separación incompleta del capital y del trabajo, ya que la gran mayoría de los duenos ocupan al mismo tiempo un puesto de Irabajador. Los pagos al trabajo son a menudo irregulares y en algunos casos parcialmente en especies.

Muchas empresas del sector informal no llegan a cumplir con toda la legislación fiscal y laboral vigente, la cual en El Salvador es muy engorrosa y compleja. Las obligaciones de recibos certilicados está limilada a las empresas con mas de 50.000 colones de ventas anuales; eslán obligadas en pagar entre el 1 y el $5 \%$ de impuestos sobre la renta las que poseen menos de 20.000 colones de capital lijo y unas ventas entre los 50.000 y los 100.000 colones; y las que declaran un capital fijo de mas de 10.000 colones deben tener los libros contables.

Por ultimo, el conceplo de esle sector implica la "tacilidad de entrada" como su caraclerística principal. Los limitados requerimientos de capilal lisico y de capital humano explica la relativamente fácil inserción de estas unidades por su relativamente bajo nivel tecnológico, aunque la escala de capilal pueda variar de acuerdo a las distinlas aclividades: en las actividades comerciales y los servicios a la persona el capilal será más limitado que en ciertas actividades manufactureras, de reparación o de los servicios de transporte público. Esta es la distinción principal entre el seclor moderno y las empresas del sector intormal y que deter- 
mina el mayor o menor acceso al crédito de las empresas.

\section{Las empresas del sector Informal}

Para llegar a cercar el universo lan helerogéneo del sector informal hay que ulilizar las cualro variables que se ha venido indicando en las líneas superiores: el nivel de educación y de capacitación profesional de los trabajadores y duehos de las "empresas", el número de empleados en estas empresas, el capilal invertido por cada una y como cuarta variable, la manera de conducir la actividad económica. Las varias deliniciones sobre las empresas que componen el sector informal, en El Salvador no concuerdan sobre los parámelros indicados.

El MiPlan define las empresas del sector informal en base a la tipología de los Irabajadores que componen el sector, el número mínimo de Irabajadores que componen una empresa y el nivel de profesionalidad del duefio o de los otros componentes. Se llega entonces a la definición siguiente. Los trabajadores del sector informal son aquellos, indica el MiPlan que generan ingresos en micro o pequehas empresas con menos de 5 empleados y en ocupaciones que no requieren un nivel educativo elevado o especialización.

Sin embargo no concuerdan sobre el monto del capital invertido en la actividad. El Minislerio de Planificación indica que una micro-empresas se deline en base a un monlo de capilal invertido de hasla los 100.000 colones. El Banco Central de Reserva considera que esle capital invertido puede llegar hasla un monto de 500.000 colones.

Para el Programa de Promoción a la pequeña y micro-empresa, PROPEMI, de FUSADES, la micro-empresa es aquella que tiene un activo fijo menor de 100.000 colones y la pequena empresa aquella cuyos activos llegan hasla 500.000 colones y hasla 10 empleados. Para la Federación de Cajas de Crédito, FEDECREDITO, la micro-empresa es aquella cuyo aclivo no supera los 100.000 colones y la pequefa aquella con activos hasta 750.000 colones.

La definición que se propondría adoptar para identificar a las empresas del sector inlormal es como sigue: que las micro-empresas son empresas básicamente familiares, con un único dueño, con un capital invertido hasta 50.000 colones y con menos de 5 empleados.

\section{Las activldades del sector Informal}

Las aclividades del sector se encuentran como jerarquizadas según la incidencia de la competencia, los niveles de capacitación y de requeri- 
miento de capilales de inversión. A primer eslabón de la pirámide encontramos los servicios personales (lustrabotas, planchadoras, lavadoras) y el comercio ambulante. Vienen después las actividades caseras (producción de alimemtos, confección de ropas, la reparación de zapatos y radios, y gran parte del pequefio comercio llevados a cabo en kioscos y pulperlas conectadas con las residencias del duefio). Siguen las actividades que requieren un taller separado y una inversión mayor para el local, herramientas y equipo más cosloso, como reparadores de electrodomésticos, carpinterlas, y producción de muebles sencillos.

El último eslabón de esla jerarqula podría ser ocupado por actividades que requieren niveles relativamenle allos de calificación o montos relativamente altos de inversión como electricistas independienles o tabricantes de productos de la metalmecánica o reparadores de automóviles, taxistas, autobasureros, etc. ${ }^{2}$

Estas micro-empresas representan el 94 por cienlo de lodas las empresas nacionales salvadorenas, por decir de su importancia relaliva. Según esludios, en El Salvador este seclor puede ser subdividido lodavia en tres niveles en base al grado de educación. El primero, el nivel mas bajo, son las actividades "ambulatorias" donde los participantes tienen un nivel de educación muy bajo en general y una inversión por debajo de los 5.000 colones. El siguiente, comprende las actividades dichas "caseras": estas no requieren de un nivel educalivo muy elevado y el capital varia entre los 25.000 colones en las actividades comerciales y $\mathbf{5 0 . 0 0 0}$ colones en la industria. El tercer nivel comprende aquellas actividades que se ejeculan en talleres afuera de la casa y por sus características registran un capital invertido hasta 50.000 colones y un nivel medio de educación

Aproximadamente el $60 \%$ de las micro-industrias pertenecen a la rama textil, con la casi lotalidad de ocupados de sexo temenino, y de la rama de la zapateria, para la producción y la reparación de zapalos donde la casi totalidad de los ocupados son hombres. Las otras ramas de la producción muy importantes para el sector son las de la carpintería y de la transformación del hierro.

\section{El empleo y los Ingresos en el sector Informal}

En 1978 el número tolal de empresas era de 60.047 unidades y daban empleo a 194.524 Irabajadores. De estas empresas, 56.464 em-

2. Estas clasificaciones han sido definidas en base a un detallado estudio que la ONG salvadoreña FINCA ha establecido para el año 1985. 
presas eran consideradas micro-empresas, representando el $94 \%$ del total y ocupando el $43 \%$ de la población económicamente activa con un promedio de 1 a 2 empleados por empresa.

Hablando de los tres sectores de la producción donde existen microempresas, el comercial reunia el $64 \%$ del tolal con 38.108 unidades 10 cual indica que es esle sector el que mas atrae las iniciativas por su "facilidad de acceso" y el capital de inicio muy limitado aunque el salario promedio por lrabajador, 304 colones mensuales, es el salario más bajo de los tres sectores. (Ver cuadro siguiente).

El sector industrial representa por su parte el $15 \%$ con 8.786 y el de servicios el $16 \%$ con 9.579 unidades. Los dos otros sectores, transporte y construcción contaban únicamente con pequenas empresas de más de cinco empleados y medianas y grandes empresas.

Los ingresos de los trabajadores del sector informal son en general más bajos que en el sector moderno, por causa de la más baja productividad del primero. Cálculos hechos en El Salvador en 1974 indicaban una diferencia entre el 34 y el $64 \%$, dependiendo del subsector. Esto se observa en la industria donde la inversión en capital es importante. El porcentaje disminuye en el sector de la construcción donde los niveles de salario se encuentran iguales en los servicios de reparación y personales. $^{3}$

En base al Censo de 1979, los niveles de salarios se habian deteriorado de por mucho. Los pagados en el sector inlormal eran iguales al $4 \%$ del salario pagado en el sector modemo y en los otros dos sectores entre el $11 \%$ (servicios) y $10 \%$ (industria). Este nivel relativo muy bajo de los diferenciales de salarios es debido a la dilerencia muy grande entre las productividades, valor de la producción por obrero (en el sector industrial la productividad de las micro-empresas represenla el $17 \%$ de la oblenida en el seclor moderno) y el valor agregado, valor agregado por obrero, (en el mismo sector, el de las micro-empresas represenla el $20 \%$ del sector moderno).

Un estudio de FUSADES, en base a las encuestas de Hogares de propósito múltiples hechas por MiPlan en 1988 y 1989 en las áreas urbanas del pais indica que la situación habia empeorado sensiblemenle desde 1978. El número de micro-empresas se habia multiplicado por 6 empleando a más del $58 \%$ de la población económicamente activa y su distribución era por su mayoría en el comercio (40\%), en el sector de los

3. Cambio y polarización ocupacional en Centro América, PREALC/OIT, EDUCA 1986, San José, Costa Rica, pag. 116 y siguientes. 
servicios i $9 \%$ ), y en la industria (20\%). A eslas se afiaden micro-empresas de iransporte $(7 \%)$ y de la construcción $(5 \%)$.

\section{La problemática del crédlto del sector Informal}

Lo que impide el crecimiento y el consecuente desarrollo del sector comenlado es el acceso al crédilo y esto por Ires molivos. El primero es porque los productores del sector informal no ofrecen ninguna garanlía financiera "clásica", como inmuebles, cuenta bancaria o capital fijo ya instalado y económicamente valorable. De esta manera, los micro-empresarios deben buscar su capilal de trabajo en el mundo de los usureros donde las lasas de interés es entre el $10 \%$ por mes hasla el 5 y el $10 \%$ por día y quizás más.

E! segundo motivo es la falta de interés por parle de las instituciones finar,ieras hacia el sector y a pesar de disponer de programas de crédito con tasas prelerenciales para el sector informal, se niegan a promover estas líneas considerando que los monlos unitarios de los crédilos son demasiado bajos, que el número de préstamos es demasiado allo y que las tasas de interés ofrecidas no son remunerativas para los servicios ofrecidos.4

La gran necesidad de crédito por los agentes de esle sector viene del hecho que el mercado del sector informal es allamente competitivo. Estos productores se ven forzados atraer los clienles vendiendo a crédilo, mientras ellos no obtienen crédito de sus abastecedores y están por lo tanto obligados a pagar su equipo y su materia prima al contado.

En 1990, las cualro Instituciones que distribuian crédito a las microempresas, han llegado a distribuir 6.814 créditos o sea han alcanzado al $1.76 \%$ del tolal del universo con un promedio de 8.273 colones por cada uno. ${ }^{5}$

\section{Los ocupados del sector Informal y su formaclón técnico profe- slonal}

La caracteríslica de la formación lécnico prolesional y de las capaci-

4. Conversaciones con algunos directores de bancos salvadorøños.

5. Ibidem que nota precedente.

6. Esta no es una particularidad de las empresas del sector intormal en EI Salvador, ni la inexistencia de una ley orgánica de la formación profesional que obligarla el sector patronal a respetar y adecuar los escalatones de salarios a las habilidades técnicas adquiridas por los obreros y técnicos profesionales, sea por certificados de aptitud sea por ancianidad en el puesto. 
dades profesionales de los nuevos y anliguos empleos en este sector se adquiere básicamenle por aulogeneración de los puestos de trabajo, dentro de las posibilidades ofrecidas por el mercado para producir y vender un producto que genere algún ingreso."

En este seclor encontramos cuatro tipos de personas ocupadas. Por una parte se encuentran los empleados que fueron despedidos y que no pueden darse el lujo de quedarse sin ingreso, en la espera de un nuevo empleo en el sector moderno. Eslos se ocupan en trabajos marginales (lustrabotas elc.) o en empleos donde puedan oblener una nueva calificación (talleres o comercio);

Por otra parte el sector informal cuenta con integranles estructurales que se comportan como verdaderos empresarios, como por ejemplo los dueños de micro-empresas informales de manulacturas y de reparación. El lercer grupo está constiluido por los jóvenes licenciados universitarios y bachilleres de escuelas lécnicas que no han todavia encontrado un empleo en el sector moderno. El último grupo está constituido por la gran masa de emigrantes del campo, quienes expulsados de las actividades agricolas llegan al mundo urbano en busca de mejores situaciones financieras.

En conclusión este sector constituye una fuente importante de empleo para los trabajadores, hombres y mujeres, jóvenes y viejos. En 1980 , este seclor daba ocupación al $49 \%$ de la población económicamenle activa nacional. Este sector ya no funciona unicamente como momento de pasaje hacia el sector moderno, sino esle proporciona empleos definitivos para los nuevos trabajadores que ingresan a la vida activa o que vienen como emigranles del campo, encuentran Irabajo y se especializan en algún oficio.

A la deliciencia de capacilación técnica se anade la falta de conocimienlos gerenciales de los empresarios de este sector. El mercado de los operadores del sector inlormal eslá principalmente constiluido por los empleados de ingresos modestos quienes compran parte de sus viveres en el kiosco informal en su barrio de residencia, quienes contratan a un albañil independiente para ayudar en la construcción de su casa y por quienes ulilizan ropas de vestir y muebles hechos por productores del barrio. Los "productores" del seclor informal raramente

7. CEPAL, borrador sin título, sobre la formación técnico profesional en El Salvador, de la Dra. Carla Macario, Santiago de Chile noviembre 1991. Este sería parte de un estudio más amplio sobre la formación protesional en Centroamérica que no se ha publicado todavla. 
sobrepasan los limiles lerritoriales del barrio en una realidad urbana grande y por supuesto no tienen sino muy raras relaciones produclivas con empresas del sector moderno.

Un estudio recién publicado por la CEPAL sobre la formación profesional en El Salvador, llegaba a la conclusión que actualmente "la oferla de formación profesional por parte del Eslado [...] no es relevante para el sector productivo del país".7 los esfuerzos de la capacitación profesional son actualmente dispersos y varias instituciones privadas han tratado de llenar el vacío que ha dejado el Eslado.

El Estado ha considerado siempre que la formación prolesional se limitaba a cursos de habilitación de la mano de obra y dejaba el aprendiz a la buena suerte de la empresa que queria hacerse cargo de esla tarea. Esta es su polílica hasta hoy en día. En el pasado los aprendices asimilaban el olicio observando y con pequenas prácticas, sin percibir ninguna remuneración duranle este largo periodo y cuando "se le consideraba medio operario, recibla una relribución inferior al $50 \%$ del salario del operario"s

En 1977 se formuló un proyecto de ley para la creación del Instituto Salvadoreño de Formación Prolesional que nunca fue disculido en la Asamblea Legislativa. De esta manera el INSAFORP fue organizado, aunque hasta hoy en dia este no tenga un estatuto legal.

Actualmente diversos ministerios disponen de un propio sistema de formación prolesional. Recordemos los Bachilleralos técnicos del Ministerio de Educación, la ENA del MAG, el ITCA del Ministerio de Educación, el (Inslituto Salvadorefio del Café) del MAG, el CENCAP siempre del MAG, elc. Mal administrados todos estos centros ulilizan el $80 / 95 \%$ de los fondos alribuidos para los salarios de los docenles y de los administrativos y del resto del personal.

Esta es la siluación de la formación profesional pública. Entre las instituciones privadas encontramos igualmenle una siluación de muy grande talla de coordinación, en donde cada una abre cursos a su aniojo con la finalidad de impartir cursos para la lormación de micro-empresarios para las personas de los barrios marginales o zonas de grande pobreza del país. Algunas de estas se han beneficiado de personal calificado y de apoyo de donaciones extranjeras.

8. Insalorp, la realidad y problemas actuales de la formación profesional ocupacional en El Salvador, jornadas hispano-centroamericanas sobre formación prolesional ocupacional, San José, Costa Rica 22/26 mayo 1989. 
Todas han creado un sistema de relaciones de amistad con pequehas, medianas y grandes empresas donde poder ubicar a los mejores egresados de sus ciclos de habilitación. El Instiluto Ricaldone ha introducido una melodología dual de capacitación en la empresa con un seguimiento que permiten tener información sobre las necesidades del personal egresado. Algunas ONG se han visto organizando módulos de capacilación lécnica en comunidades de retornados y han desarrollado curso de capacilación técnica dentro de las muchas actividades ligadas al desarrollo de zonas del pais.

Se puede compartir la opinión de la CEPAL que en EI Salvador, en general, "no existe actualmente una política nacional de formación profesional... Una parte importante de la capacitación se lleva a cabo directamente en las empresas... y... los programas de entrenamiento que existen son el resultado de los estuerzos dispersos de distintas organizaciones sin coordinación... Es indispensable la creación de una institución que cumpla el rol de coordinación y de reclorla de la formación profesional...

Se necesita de una política nacional donde los procesos de ensefanza/aprendizaje se lleven a cabo con recursos adecuados y que entrente una práctica de descentralización administrativa y de las práctica pedagógicas, en coordinación estrecha con el mundo del trabajo, sindical y empresarial para que la formación sea adecuada a la nueva oferta de empleos.

\section{Concluslones actuales}

Es ahora un hecho que la larga crisis de la economia mundial ha provocado un lenlo proceso de "inlormalización" de las economias de los paises tecnológicamente dependientes. El conflicto armado que ha venido sacudiendo la sociedad salvadorena ha frenado el ritmo de inversiones de capitales desde 1975 y agudizado los efectos de esta crisis. Las estadísticas de la distribución de la PEA salvadorena entre los sectores modemo e informal lo explican bien claramente.

Considerando que la economla nacional es una única se tendrá que definir los roles de cada seclor de la producción. En este panorama hay que modernizar el sector inlormal para que se integre complelamenle en las ramas respeclivas. Para esto falla establecer condiciones para que el seclor moderno, privado y estatal subcontrate directamente a microempresas del sector inlormal. Falta una legislación adecuada para reforzar sus actividades, una política de crédito de apoyo a la modernización de sus lecnologlas productivas, una política impositiva de 
formento del sector y una estralegia particular al sector informal para la formación protesional.

La rápida expansión del sector informal urbano observada por todos los estudios en la última década en El Salvador viene de una profunda crisis que ha venido afectando los sectores nacionales de la producción industrial, agro-industrial y del más amplio sector ganadero. Esta crisis se encuentra a la base de la emigración masiva del campo hacia las ciudades y hacia el exterior del país y ha sido relorzada por el conflicto armado.

La modernización del agro no ha llegado a las fincas pequehas, donde se concentra la gran mayoría de los campesinos y de donde sale la producción nacional de cultivos de granos básicos. Además los propietarios de las ya limiladas parcelas la parten para dar tierras a sus hijos y este fenómeno lleva el empobrecimiento de las tierras porque las superficies son insuficientes para cultivar, no se pueden respelar los descansos lo que lleva a una consecuente disminución de la productividad agricola justo en esos cultivos esenciales para la vida colidiana.

Cuadro sobre la variación del porcentaje de ocupados en los seclores formales e informales por categoría ocupacional

\begin{tabular}{|c|c|c|c|c|c|}
\hline & 1978 & 1990 & & 1978 & 1990 \\
\hline \multirow{2}{*}{$\begin{array}{l}\text { Tot. Secl. Formal } \\
\text { Patronos } \\
\text { Proles. Indep. }\end{array}$} & $\begin{array}{r}60.40 \\
1.00\end{array}$ & $\begin{array}{r}49.20 \\
1.20\end{array}$ & Tot. Sect. Inform. & $\begin{array}{r}39.60 \\
\ldots\end{array}$ & 50.80 \\
\hline & 090 & 0.70 & $\begin{array}{l}\text { Trabajadores } \\
\text { por cuenta propia* }\end{array}$ & 24.60 & 44.70 \\
\hline $\begin{array}{l}\text { Empleados y Obr. } \\
\text { a sueldo tijo }\end{array}$ & 43.40 & 36.70 & ......... & & \\
\hline $\begin{array}{l}\text { mpleados y Obr. } \\
\text { destajo }\end{array}$ & 15.10 & 9.70 & & & $\ldots$ \\
\hline $\begin{array}{l}\text { Familiares sin } \\
\text { remuneración }\end{array}$ & .. & 0.80 & $\begin{array}{l}\text { Familiares sin } \\
\text { remuneración }\end{array}$ & 5.50 & 6.10 \\
\hline Domésticos & & & Domésticos & 9.50 & $\ldots$ \\
\hline
\end{tabular}

- en los trabajadores por cuenta propia del sector intormal en 1990 se han incluido los trabajadores domésticos.

Fuente:: Encuestas de hogares de propósitos múltiples de 1978 y 1990, Miplan, San Salvador.

Los procesos inflacionarios que han golpeado el pais han provocado aumentos también en los precios de los productos alimemarios, mientras 
que los salarios mínimos reales calculados a precios constantes de 1970 por el PREALC-OIT, disminulan sin parar, pasando de 72 colones mensuales en 1965 hasta 43 colones mensuales en 1983. Mientras el salario minimo real de la industria variaba de 165 colones mensuales en 1965, 193 colones en 1970, 180 colones en 1980 y 133 colones en 1983. Siempre siguiendo las observaciones del PREALC-OIT, en 1980 únicamente el $24 \%$ de la población rural podía satislacer las necesidades básicas con su trabajo."

A estos factores económicos estructurales podemos anadir factores de lipo sico-sociales como la ampliación de los productos de consumo disponibles y la imagen de la vida social urbana llevada por las capas medias, son otras condicionantes a la base de la emigración masiva de la población rural hacia la capital del país, San Salvador. Esta nueva población va engrosar las filas de los subempleados del sector informal.

\section{Hipotesls de centros artesanales populares}

Los análisis precedentes del sector informal y de la sintesis de las aclividades de lormación profesional nos indican que la actual practica de producir produclores calificados destinados a iniciar micro-empresas no ha producido el tejido de empresas en donde se desarrolle una economia nacional. Habrá que hacer un esfuerzo para producir las herramienlas que permitan llegar a un esquema tentativo de formación prolesional adecuado a las necesidades de los procesos productivos existentes en el pais.

Un artículo escrito por el padre Ibizale de la Universidad Centroamericana "J.S.Canas" de San Salvador en 1987, indicaba que una hipólesis podia partir de la estructura de la demanda familiar e industrial. De esta se podian deducir las necesidades nacionales y plantear las producciones nacionales que se podrian emprender para satisfacerlas, en base a las tecnologías existentes, disponibles y razonablemente accesibles. De alli, calculando el "efeclo sobre el empleo" se llegaría a una definición general de las necesidades en formación profesional y crear los curriculum adecuados a la realidad de El Salvador. ${ }^{10}$

Considerando la misma metodologia, se ha aplicado el modelo a una realidad campesina rural. Se Iralaba de establecer un modelo micro-

9. PREALC/OIT. op. cit.

10. Boletín de Ciencias Económicas y Sociales, julio/agosto 1987, año X, NO. 4, Universidad Centroamericana José Simeón Cañas, San Salvador, El Salvador, p. 226-252. 
económico de creación de empleos en una zona dada del país. Eslamos seguros que este modelo podrla ser repelido a nivel naclonal. Sin embargo no se llegarlan a identiticar las necesidades especilicas regionales (micro-localidades o espacios económicos más amplios), que son las bases para el desarrollo espacial de este pals.

Se ha entonces creado un modelo basado en esta hipótesis pero quedándonos a un nivel local con dalos tomados de una encuesta de ingresos de 109 familias campesinas llevada a cabo este ano en las zonas rurales del Depto. de Chalatenango, en las zonas de La Palma, y centro/orientales del departamento.

Con los dalos oblenidos se ha llegado al establecimiento de un balance lamiliar promedio, para poder establecer la base de una canasta básica. Los gastos anuales por familia obtenidos en base a las declaraciones de los jefes de familias entrevistados, en general y para todo el departamento son de 11.126 colones al ano. Los ingresos anuales monetarios por familia obtenidos por la misma encuesta, son en promedio de 11.872 colones al ano.

Se establece que existe una potencialidad de 1.121 .734 colones al ano, o sea 102.278 colones que buscan repartirse enlre grupos de bienes disponibles." Las familias entrevistadas se supone busquen los bienes en la inmediata cercania de sus hogares, y cuando no los encuenIran los buscan en los mercados disponibles con costos de transporte considerables por las débiles economias familiares. Se ha entonces establecido un modelo de estructura de la demanda rural, calculando el porcentaje promedio que la familia destina a estos bienes.

Se ha entonces consiruido un modelo de la repartición del gasto del conjunto familiar que puede ser considerado como un indicador de la situación en que viven las familias. Los dos grandes primeros rubros de esla canasla son: la alimentación y el vestuario. El tercer rubro es la salud con sus gastos afines. Viene el transporte y luego la educación. Los enseres de la casa y los demás gaslos preocupan poco a los chalalecos, que ha sido la población base de nuestra encuesia. ${ }^{12}$

11. Las diferencias entre los ingresos y los egresos son tan limiladas que se puede constatar que no hay ninguna capacidad de ahorro por parte de estas familias. Su equilibrio es entonces precario y a la merced de cualquier catástrole o accidente natural. Además los ingresos son tan reducidos que aunque hubiera algún ahorro, no sería en todo caso suticiente para compensar la tasa de inflación de la moneda nacional.

12. Boletín de Ciencias Económicas y Sociales p. cit. La estructura de la de- 
La repartición del gasto del conjunto de familias considerado es la siguiente: (en porcentajes del gasto total)
a. Alimentacion
40.30
b. ropa y zapatos
28.89
c. bienes para el hogar (muebles, vasos y platos, eléctrodomesticos)
d. educación (cuadernos, lápices, unilormes)
5.04
e. salud (medicinas y consultas)
9.02
f. gastos de transporle
9.81
g. otros gastos
2.51

En una óplica de iniciar talleres artesanales, podemos considerar los espacios que exislen para la creación de nuevas actividades de producción en referencia a cada nubro de gastos delinidos en la estructura delinida. ${ }^{13}$

Los dos primeros son evidentemente los rubros de alimentos y de prendas de vestir. Los alimentos elaborados simples, los zapatos y prendas de vestir que tienen un potencial del casi $70 \%$ del tolal $(69.19 \%)$ del gasto familiar. Esle es igual a una suma igual a 487,686 colones para el mismo conjunto de 109 tamilias que se considero en el estudio. Se puede anadir aquí, también el gaslo en los unilormes escolares que son importantes en una familia promedio de 6.65 miembros.

Panaderías, dulcerias, molinos de nixlamal, pequenas y bien abastecidas tiendas de caserio, pollos, huevos, leches, quesos, mieles, productos de la horticullura y olras actividades pueden ser encontradas. La variedad de alimentos preparados puede ser investigada lambién para su "exporlación", más allá de los límiles municipales. Un programa de asislencia a la nutrición familiar permitiría introducir anliguos platos y manjares reorientando gastos y volviendo a introducir productos nacio-

manda familiar que el Dr. Ibizale ha establecido para San Salvador (o zona urbana) bien se podrla aplicar para una misma hipotesis de trabajo en zona urbana.

13. Boletin de Ciencias Económicas y Sociales op. cil p. 233. La estructura de la demanda establecida por el Dr. Ibizate aparece diferente a la estruclura indicada en este estudio. El único rubro que se encuentra similar es el de la alimentación. La vivienda aparece en el segundo lugar onn $27 \%$ del gasto, el transporte con $5 \%$, elc. Estas diferencias en la distribución interna se pueden atribuir a una diferente distribución de los rubros de gastos en zonas urbanas $y$ en las zonas rurales. 
nales.

Los sastres y costureras ya ocupan parte de este espacio, sin embargo se puede hacer espacio para la labricación de telas. Los zapateros tienen trabajo. pero también lo podrlan tener los curtidores del cuero. Con un mejor material la talabartería tiene un espacio local, nacional e internacional, como el mercado centroamericano. Estos grupos de actividades llevan consigo un número importante de empleos ligados a la distribución.

El grupo de los muebles y allarería hogarena ocupa el $4.39 \%$. Con las dificultades de conseguir las materias primas, (la madera en principio no es abundanle en la zona de Chalatenango pero más que en otras partes del pais), las necesidades se deben haber reducido, por lo lanto la demanda de eslos bienes se encuenlra limitada. Aunque enconlrando materiales que reemplazen la madera, la demanda de estos bienes queda insatislecha.

La affarería se entrenla a la competencia de pláslicos baralas. La culpa lo tiene las lécnicas de cocción del barro y los mismos materiales de base que no son como lo suficientemente resistentes y que no llevan a un producto lo sulicienlemente acabado para el uso colidiano. Hay campo para artesanos alfareros y otras actividades afines con la consecuente cola de vendedores y transportistas.

Los gaslos en medicinas dejan algún lugar a una larmacopea más nacional y con plantas medicinales nacionales. Es probable que parte de estos gaslos puedan ser desviados primero con obras de protección ambiental que reduzcan las enfermedades, después con medicamenlos y medicinas producidas con planlas medicinales locales y nacionales y después en olros tipos de gastos no incluidos en esta estadística.

Los gastos de Iransporte son necesarios para movilizarse en largas distancias en el campo. Pero eso es signo que existe una polencialidad para los artesanos ligados a las reparaciones de los medios de transporte, como mecánicos, mecánicos de banco y soldadores, elc. Aquí los servicios de reparación de sillones en cuero y en lelas de los vehículos dispone de un mercado polencial.

La segunda etapa del modelo es el cálculo de inversión por cada producción identificada y en función de las disponibilidades establecer las prioridades o las concentraciones de los procesos produclivos necesarias por economias de escala. La base del gaslo existentes sumada a los gastos adicionales producidos por la multiplicación de los ingresos producidos por los nuevos emprendedores indicará la capacidad de gasto total disponible para un cierlo número de produclores nuevos que se 
podría inslalar.

La elaboración de un modelo de creación local de empleo está lodavia lejos de ser lormulada. Fallan estudios sobre las eslructuras de la demanda locales y regionales, establecer las elasticidades por cada rubro y hacer las necesarias proyecciones sobre las oportunidades de crear capacidades profesionales correspondientes a la satisfacción de dichas demandas.

\section{Esta es la propuesta}

Sin embargo se puede afirmar que esta es una dirección para la organización de la economia nacional que busque su crecimiento en la satislacción de las necesidades internas. Es en base a eslas primeras premisas, que habría que organizar un modelo de simulación económica y construir un conjunto de cadenas de producciones que nos lleve a la construcción de un cuadro de insumo produclo indicador de las interrelaciones entre ramas y sub-ramas.

Esle trabajo nos ensenaría los cuellos de bolella inevitables dada la esiructuración dependiente de esta economía, y permitiría determinar los "by-pases" a crear, cuando se pueda, con producciones nacionales y locales para que se llenen los vacíos existentes actualmente entre las diversas sub-ramas. Aqui no se trala de evadir las "leyes" de un mercado de libre empresa, sino de ubicar aquellas actividades que bien pueden ser asumidas por nuevos inversionistas e identificar la escala de inversión actualmente soportables por eslos nuevos empresarios, posiblemente del sector informal.

En conclusión, este ejemplo puede ser retomado en cualquiera de los departamentos del país (y también en olro rubro de la producción como las hortalizas u otro), para establecer la repartición de los gastos de las familias según rubros de gasto y estos pueden ser valorados, de manera que se encuentre una base más cercana de la realidad para la creación de Centros artesanales que satisfagan la demanda inmediata y que dispongan de una clientela potencial lo sulicientemente amplia para garanlizar la existencia económica de los nuevos productores de bienes de consumo populares. Este modelo podría al linal frenar la migración del campo hacia la ciudad garanlizando salidas económicas a los productores establecidos. 